\title{
Susceptibility to pressure neuropathy distal to a constricting ligature in the guinea-pig
}

\author{
T SHIMPO, R W GILliATt, R P KENNETT, P J ALLEN \\ From the University Department of Clinical Neurology, Institute of Neurology, Queen Square, London, UK
}

SUMMARY Silk ligatures were tied round the sciatic nerve in guinea-pigs and left in place in order to produce persistent nerve constriction. Serial nerve conduction studies over the following 9 weeks showed a reduction in motor conduction velocity distal to the ligature. The presence of axonal atrophy in tibial nerve fibres in the leg was subsequently confirmed by histological studies. These changes were not seen in a second group of animals in which similar ligatures were tied but removed after 6 hours. When the ligatures were left in place, the animals developed local plantar nerve lesions in the sole of the foot on the affected side, which were thought to be due to pressure from the floor of the cage. Local pressure changes of varying severity were seen in the foot in all the constricted nerves, but were only occasionally found in control nerves from the opposite foot, or in nerves which had been constricted for a few hours by ligatures which were then removed. These results suggest that atrophic nerve fibres distal to a persistent constriction may be particularly sensitive to local pressure.

Earlier experiments on guinea-pigs have shown that constriction of the sciatic nerve by a silk ligature in the thigh may be followed by distal axonal atrophy. ${ }^{1}$ For this to occur, it was necessary to tie the ligature tightly enough to cause Wallerian degeneration of most of the large myelinated fibres. When this was done, surviving fibres in the tibial nerve distal to the ligature were found to have a reduced external diameter in relation to internodal length, and a reduced axon/external diameter (g) ratio. Maximal conduction velocity in motor fibres to the plantar muscles was reduced in the leg distal to the ligature, but it was not possible to decide how much of this reduction was due to the degeneration of large fastconducting fibres, caused by tying the ligature, and how much to the atrophic change in surviving fibres.

In the present study a constricting ligature round the sciatic nerve in the thigh has again been used to produce distal axonal atrophy in the guinea-pig. In a separate group of animals a similar ligature was tied but removed after 6 hours, so that the effects of tying the ligature could be distinguished from those of pro-

Address for reprint requests: Professor R W Gilliatt, University Department of Clinical Neurology, Institute of Neurology, Queen Square, London, WCIN 3BG, UK.

Received 20 February 1987 and in revised form 20 May 1987. Accepted 22 May 1987 longed constriction. When studying conduction in motor fibres to the plantar muscles, particular attention was paid to conduction in the foot itself, which revealed changes that had not been noted in previous experiments.

\section{Methods}

Experiments were carried out on guinea-pigs aged 3-5 months. The animals were kept in individual cages with solid floors covered by a layer of sawdust $1-2 \mathrm{~cm}$ in depth.

Motor conduction studies were carried out under general anaesthesia before and at 1 or 2-week intervals after tying a ligature round the sciatic nerve in the upper thigh. Stainless steel needles placed close to the tibial nerve in the thigh and at the ankle served as stimulating cathodes. The upper cathode was $1 \cdot 5-2 \mathrm{~cm}$ distal to the site of ligature; conduction distance between the two sites of stimulation ranged from $3.5-5 \mathrm{~cm}$, and the distance from the distal cathode to the muscle was approximately $3 \mathrm{~cm}$. Recording from the plantar muscles was through subcutaneous needles, one placed transversely over the muscles and the other distally. Supramaximal shocks were used; evoked muscle action potentials (MAPs) were amplified and displayed on a Medelec MS6 electromyograph, and recorded on light-sensitive paper using a fibre-optic recorder. On some occasions an additional cathode was placed close to the medial plantar nerve in the proximal part of the sole, approximately $2 \mathrm{~cm}$ from the active recording electrode. MAP amplitude was measured from the peak of main negative deflection to the peak of the following positive deflection of the action potential. 
For the measurement of MAP area, only the main negative deflection of the action potential was used.

For anaesthesia intramuscular ketamine $(30-40 \mathrm{mg} / \mathrm{kg})$ was given with xylazine $(5 \mathrm{mg} / \mathrm{kg})$ and repeated when necessary. To maintain body temperature while recording under anaesthesia, animals were covered in cotton wool. Skin temperature was monitored over the calf muscles and varied from $33^{\circ}-36^{\circ} \mathrm{C}$.

In each animal a silk ligature (Ethicon-braided $0.2 \mathrm{~mm}$ gauge) was tied round the sciatic nerve in the upper thigh on one side, under general anaesthesia and with aseptic precautions. The tightness of the ligature was determined by experience, the object being to tie it tightly enough to cause Wallerian degeneration in most of the large myelinated fibres. In one group of animals the ligatures were left in place, but in another group of animals the wound was reopened under anaesthesia 6 hours after the first operation, and the ligature removed. To estimate the amount of Wallerian degeneration caused by the ligature, the plantar MAP evoked by supramaximal nerve stimulation at the ankle was recorded after 1 week, and compared with the pre-operative recording. Animals with a reduction in MAP amplitude of more than $60 \%$ were then used for serial studies. A few animals in which ligatures had been tied tightly enough to cause complete loss of the plantar MAP were also followed, together with some in which nerve crush was carried out instead of ligature, so that the time-course of regeneration from the upper thigh could be followed without the complication of surviving motor fibres being present.

At the end of the physiological experiments animals were killed by the intracardiac injection of pentobarbitone, and portions of the tibial and medial plantar nerves removed from both sides, care being taken to avoid any change in the length of the specimen. After fixation in $4 \%$ glutaraldehyde in Sorenson's buffer at $\mathrm{pH} 7 \cdot 4$, the specimens were divided into portions for teasing and for transverse sections. After post-fixation in $1 \%$ aqueous osmium tetroxide, the subsequent preparation of semithin transverse sections and of single teased fibres was carried out using methods identical with those described previously. ${ }^{2}$

Quantitative measurements were made on photographic prints of semithin transverse sections at a final enlargement of $\times 2,850$. Axon and myelin perimeters were traced on a digitising pad, and the corresponding values for axon and total fibre area were calculated, using a Research Machines $380 \mathrm{Z}$ computer. As in previous studies, the values for axon and external fibre diameter were not obtained by direct measurement but were computed in each case from the crosssectional area of the axon or fibre, the computed diameter being that of a circular profile having the same area as that measured.

\section{Physiological results}

There were eight animals in which a ligature had been tied round the sciatic nerve in the thigh and left in place, and in which distal nerve stimulation at 1 week revealed a fall in amplitude of the plantar muscle action potential (MAP) of more than $60 \%$ (mean fall in amplitude $85.4 \%$ ). These animals (group A) were matched with a second group of eight animals (group
B), in which the ligatures were removed after 6 hours, and in which distal nerve stimulation at 1 week showed that the amplitude of the plantar MAP was reduced by a similar amount (mean fall in amplitude $85 \cdot 2 \%$ ). In all 16 animals motor nerve conduction studies were carried out 1, 3, 5, 7 and 9 weeks after ligature, so that changes in motor conduction velocity (MCV) in the leg, distal motor latency (DML) in the foot, and MAP amplitude and area could be followed.

Results for MCV and DML are summarised in fig 1. During the first week after ligature MCV between knee and ankle fell from pre-operative mean values of 43.7 and $41.1 \mathrm{~m} / \mathrm{s}$ to 33.4 and $32.4 \mathrm{~m} / \mathrm{s}$ in groups A and $B$ respectively. There was no difference between the two groups at 3 weeks but thereafter velocity in group B gradually increased, the mean value reaching $42.6 \mathrm{~m} / \mathrm{s}$ by 9 weeks, while the mean velocity for group A animals remained low $(34 \mathrm{~m} / \mathrm{s}$ at the end of 9 weeks). When individual values for MCV at 5, 7 and 9 weeks were pooled, the difference between the means of the group A and B nerves was significant at the 0.01 level (table 1). The morphological changes which might be responsible for the initial fall in MCV in group A and B nerves, and for their significantly different subsequent behaviour, will be discussed in a later section.

In group B animals the mean DML in the foot showed a small increase in the first week after ligature, followed by a partial return to the pre-operative value. In group A there was a similar increase in DML at 1 week; in two animals high values (greater than $3 \mathrm{~ms}$ ) were recorded later in the 9 week period,

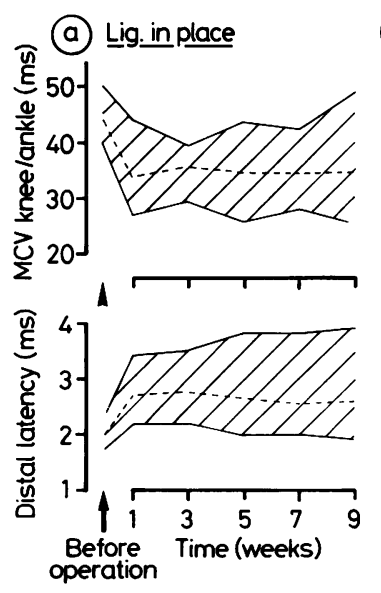

(b) Lig. released

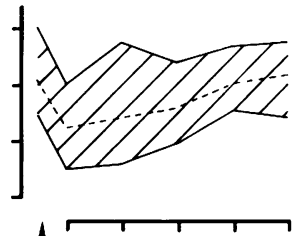

$\Lambda$

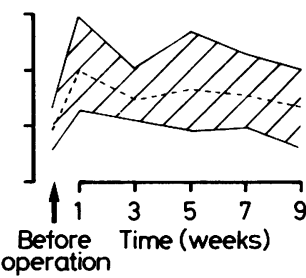

Fig 1 Mean and range for $M C V$ in the leg (above), and $D M L$ in the foot (below), before operation and at 1, 3, 5, 7 and 9 weeks afterwards, in group $A$ and group $B$ animals. 
Table 1 Mean values and SD for MCV, DML, and MAP amplitude and area, for affected and control nerves of group $A$ and $B$ animals, before and after unilateral nerve ligature. (Number of observations shown in brackets)

\begin{tabular}{|c|c|c|c|}
\hline & Before operation & 1 and 3 weeks & 5,7 and 9 weeks \\
\hline \multicolumn{4}{|l|}{$\mathrm{MCV}(\mathrm{m} / \mathrm{s})$} \\
\hline A (lig) & $43 \cdot 70 \pm 3 \cdot 50(8)$ & $34 \cdot 40 \pm 4 \cdot 30(16)$ & $34.00 \pm 6.90(24)$ \\
\hline B (lig released) & $41 \cdot 10 \pm 4 \cdot 80(8)$ & $33 \cdot 30 \pm 5.80(16)$ & $39 \cdot 50 \pm 5.40(24)$ \\
\hline control & $42 \cdot 70 \pm 6.90(16)$ & $46.00 \pm 6.80(32)$ & $49.50 \pm 6.50(48)$ \\
\hline \multicolumn{4}{|l|}{ DML (ms) } \\
\hline A (lig) & $1.96 \pm 0.18(8)$ & $2.72 \pm 0.44(16)$ & $2 \cdot 58 \pm 0.57(24)$ \\
\hline B (lig released) & $1.92 \pm 0.24(8)$ & $2.73 \pm 0.57$ (16) & $2.50 \pm 0.51(24)$ \\
\hline control & $2 \cdot 10 \pm 0.24(16)$ & $2.02 \pm 0.36(32)$ & $2.05 \pm 0.36(48)$ \\
\hline \multicolumn{4}{|c|}{ MAP amplitude (mV) } \\
\hline A (lig) & $5 \cdot 50 \pm 1 \cdot 50(8)$ & $1.20 \pm 1.20(16)$ & $2 \cdot 10 \pm 1 \cdot 40(24)$ \\
\hline B (lig released) & $3 \cdot 90 \pm 1 \cdot 10(8)$ & $1.00 \pm 0.80(16)$ & $3 \cdot 10 \pm 1 \cdot 50(24)$ \\
\hline control & $4.60 \pm 2.60(16)$ & $4.80 \pm 2.40(32)$ & $5 \cdot 60 \pm 2 \cdot 50(48)$ \\
\hline \multicolumn{4}{|l|}{ MAP area +} \\
\hline A (lig) & $1328.00 \pm 385$ & $518 \cdot 00 \pm 422(16)$ & $\dagger\{682 \cdot 00 \pm 433(24)$ \\
\hline B (lig released) & $1015 \cdot 00 \pm 355(8)$ & $419 \cdot 00 \pm 273(16)$ & $\{1004.00 \pm 451$ (24) \\
\hline control & $1271 \cdot 00 \pm 691(16)$ & $1278 \cdot 00 \pm 536(32)$ & $1428 \cdot 00 \pm 609(48)$ \\
\hline
\end{tabular}

*Significantly different by Student's $t$ test $(p<0.01)$.

†Significantly different by Student's test $(p<0.05)$

†MAP area in arbitrary units ( 1 unit $\left.=2 \times 10^{-3} \mathrm{mV} \times \mathrm{ms}\right)$.

but the mean for the group did not differ significantly from that of group B.

With normal growth of the animals during the 9 week period, MCV in the unconstricted control nerves of the opposite leg increased from a mean value of $42.7 \mathrm{~m} / \mathrm{s}$ to $49.0 \mathrm{~m} / \mathrm{s}$; DML was unchanged.

Results for plantar MAP amplitude and area are summarised in fig 2 . In each case the potential evoked by stimulation at the ankle was used for measurement. It can be seen that the initial fall in MAP amplitude and area was similar in groups A and B, but that subsequent findings were different. In spite of considerable individual variation, there was in group B substantial recovery of mean amplitude and area, the latter returning to its pre-operative level by 7 weeks. In group $A$ the recovery of amplitude and area was less complete, some animals showing virtually none. For both amplitude and area the means of pooled individual values at 5,7 and 9 weeks were significantly reduced in group A compared with group B (table 1).

It may be asked whether recovery of plantar MAP amplitude and area, when it occurred, could be the result of regeneration from the site of ligature. This seemed unlikely in view of the distance from the ligature to the muscle $(9-10 \mathrm{~cm})$, and the possibility was excluded by experiments on a separate series of animals in which the time-course of regeneration was followed after complete lesions of the sciatic nerve in the thigh. The lesions were made by nerve crush (three animals) and by ligatures tied for 6 hours, after which they were released (three animals). Nerve conduction studies were then carried out weekly. After nerve crush plantar MAPs were first evoked by nerve stimulation at 7 weeks (two animals) and 8 weeks (one animal); after ligature for 6 hours evoked MAPs were first recorded at 7 weeks (one animal) and 9 weeks (two animals). Immediately after reinnervation motor velocity was grossly reduced (less than $10 \mathrm{~m} / \mathrm{s}$ ) and distal latency was markedly increased (up to $30 \mathrm{~ms}$ ). Nine weeks after proximal crush or ligature the axonal velocities of the regenerated motor units were in the range $11-16 \mathrm{~m} / \mathrm{s}$, with distal latencies ranging from 6-27 ms. Action potentials from the recently regenerated motor units were polyphasic and of low voltage. Similar potentials were sometimes seen when recording at 9 weeks from the affected nerves of group
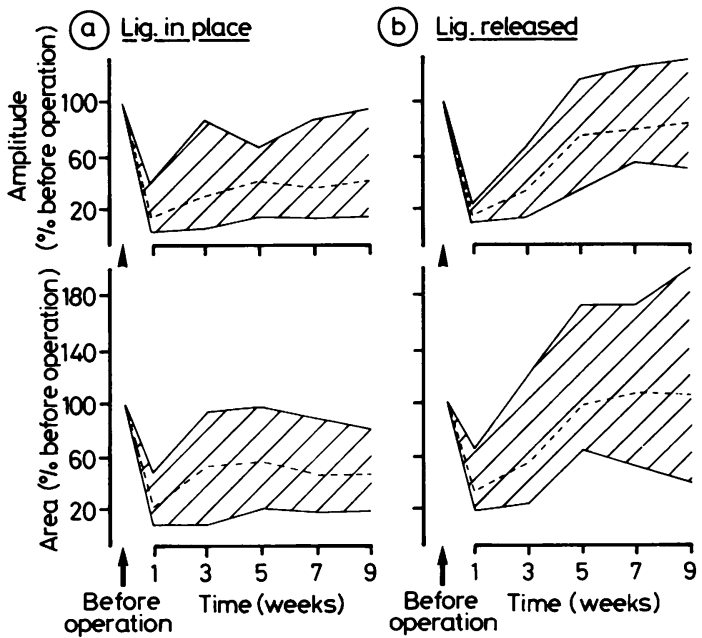

Fig 2 Mean and range for plantar MAP amplitude (above), and area (below), before operation and at 1, 3, 5, 7 and 9 weeks afterwards, in group $A$ and $B$ animals. Results are expressed as percentages of the pre-operative values. 
B animals; they could be identified by their low proximal velocities and prolonged distal latencies, which combined to separate them from the main deflection of the evoked MAP.

When recording at high amplification from affected group A nerves, low voltage components were seen with long distal latencies but with relatively rapid conduction velocities between knee and ankle. An example is shown in fig 3 , taken at 9 weeks, and it can be seen that the total duration of the muscle response was still more than $20 \mathrm{~ms}$. Two easily identifiable late components are arrowed; these had axonal velocities of 33.3 and $30.8 \mathrm{~m} / \mathrm{s}$ between knee and ankle, which may be compared with $36.4 \mathrm{~m} / \mathrm{s}$ for the earliest component of the main deflection. From the use of an additional stimulating cathode in the proximal part of the sole it was clear that the conduction delay for the late components was mainly distal to this point.

Low voltage late components with distal latencies ranging from $7-25 \mathrm{~ms}$, and with axonal velocities greater than $20 \mathrm{~m} / \mathrm{s}$ between knee and ankle, were found on the affected side in five of the eight group $A$ animals. They were sometimes seen at 5 weeks but not before this; at 7 and 9 weeks they were relatively com-
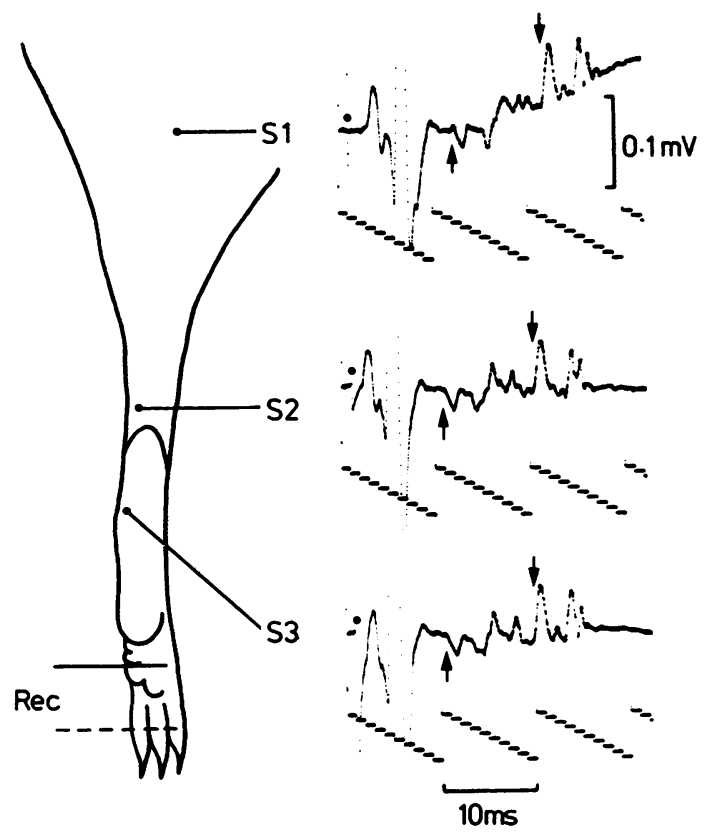

Fig 3 Plantar MAPs from the affected side of a group $A$ animal 9 weeks after ligature to show low-voltage late components. The amplitude of the main deflection of the response (not shown at this gain) was $0.7 \mathrm{mV}$. Conduction velocity in the leg was $36.4 \mathrm{~m} / \mathrm{s}$ for the main deflection; for the two late potentials marked by arrows the corresponding values were 33.3 and $30.8 \mathrm{~m} / \mathrm{s}$. mon. Only one such potential was seen on the affected side in the eight group B animals, but two examples were seen when recording from the plantar muscles on the opposite (control) side of the 16 group $A$ and $B$ animals.

This combination of prolonged distal latency and relatively high velocity in the leg, seen in some of the motor axons, particularly in group A animals, raised the possibility of local nerve damage in the foot. The appearances were similar to those seen previously in animals with pressure neuropathy of the plantar nerves from the floor of the cage. ${ }^{3} \mathrm{~A}$ characteristic feature in the previous study had been the sparing of the deep peroneal (anterior tibial) nerve supplying extensor digitorum brevis on the dorsum of the foot. Accordingly, evoked MAPs were recorded from this muscle on the affected side in two group A animals which were known to have marked changes in evoked MAPs from the plantar muscles, with temporal dispersion due to the presence of low-voltage longlatency components. In both cases the MAPs recorded from the dorsum of the foot were of normal latency and wave-form (fig 4).

\section{Histological results}

At the end of the experimental period, portions of tibial nerve just above the ankle were taken from both sides of group A and B animals. In both groups trans verse sections from the affected side showed that the number of large myelinated fibres was decreased, witho a relative excess of small fibres, some of them in regenerating clusters. A small amount of osmiophilic debris was present.

Quantitative studies were carried out on tibial nerve sections from the affected side of four group $A$ and four group B animals, and from the control nerves of the opposite side in six of them. Histograms of axon and external fibre diameter for one pair of animals are shown in fig 5 . It can be seen that in both cases the density of large-diameter fibres was reduced on the affected side. In the large-diameter fibres of the affected group A nerve there was a reduction in the axon/external diameter (g) ratio; this was not present in the corresponding nerve from group $B$. In both animals the density of small myelinated fibres was increased on the affected side, suggesting that regenerating fibres were present.

Table 2 shows mean $\mathrm{g}$ ratios for large $(7-10 \mu \mathrm{m}$ external diameter) fibres in the affected and control nerves of the two groups of animals. This diameter range was chosen to exclude any possible contamination by regenerating fibres. There was the further advantage that within this range the $\mathrm{g}$ ratio in control fibres did not vary significantly with fibre size. Table 2 shows that there was a highly significant re- 


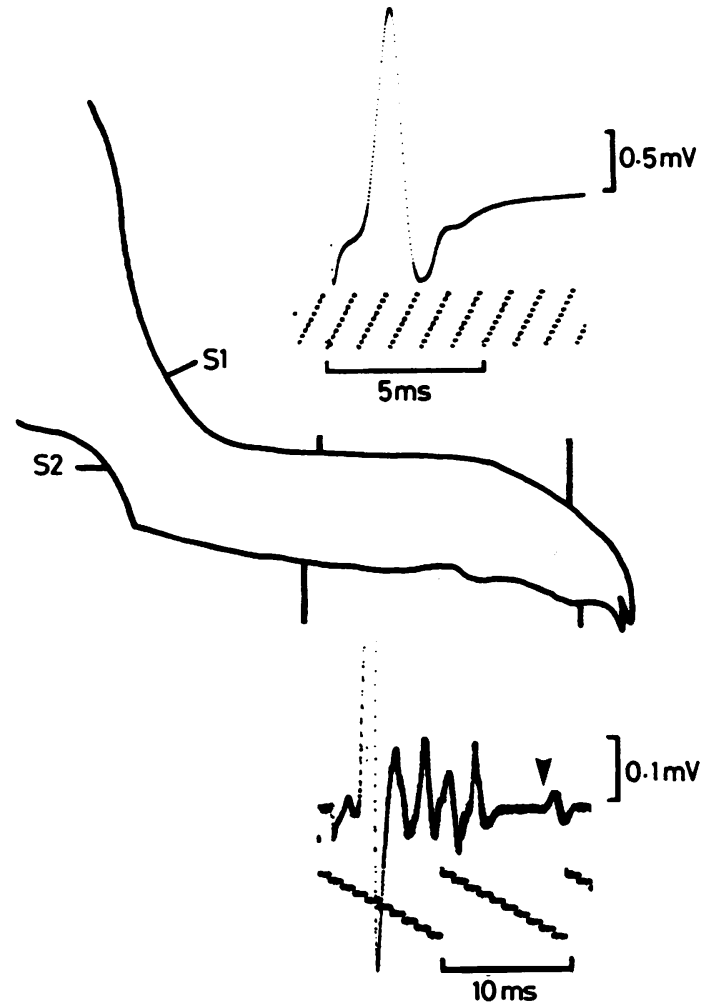

Fig 4 Records from the affected side of a group $A$ animal, 9 weeks after ligature. MAPs were recorded from $m$. extensor digitorum brevis on the dorsum of the foot (above) and from the plantar muscles (below), with stimulation of the anterior and posterior tibial nerves just proximal to the ankle. Low-voltage late components were recorded from the plantar muscles but not from $m$. extensor digitorum brevis. As in fig 3 the late components had a relatively high velocity in the leg; the longest-latency component (arrowed) had a velocity in the leg of $26.5 \mathrm{~m} / \mathrm{s}$ compared with $30.2 \mathrm{~m} / \mathrm{s}$ for the early component.

duction in the mean $g$ ratio of the affected fibres in group A compared with affected group B fibres and controls.

In teased fibre preparations from tibial nerves between the knee and ankle, surviving fibres from group $A$ and $B$ nerves showed few abnormalities. There was some loss of the normal smooth contour of the internodes in group $\mathrm{A}$, but the nodes of Ranvier appeared normal, and paranodal demyelination was rarely found.

When the medial plantar nerves were examined, it was immediately apparent in loosely teased fascicles from the affected side of group A animals that local demyelination had occurred in the sole of the foot. Seven nerves were available, and it was clear that in severely affected animals extensive demyelination and fresh Wallerian degeneration had occurred over a length of $5-6 \mathrm{~mm}$ in the centre of the sole. Over this distance the demyelination was patchy, and lesions in different fibres and fascicles were not exactly at the same level.

In single fibres the demyelination was accompanied by changes in the paranodal myelin, which was thin and tapered on the side of the node of Ranvier further from the centre of the lesion, and thickened and irregular on the opposite side. An example from a mild lesion is shown in fig 6 . It was usually the case that the asymmetry of the paranodal myelin was more obvious on the proximal than on the distal side of a lesion; some fibres appeared to degenerate at this level, loosely teased bundles showing an abrupt loss of fibres and an increase in osmiophilic debris distally.

Transverse sections were taken from the medial plantar nerve distal to the main region of demyelination described above. They showed extensive loss of large fibres and a relative excess of small thinly remyelinated axons which were thought to be regenerating, since some were in identifiable regenerating clusters. Larger axons without a myelin sheath were only occasionally seen.

The severity of the plantar lesions varied in group

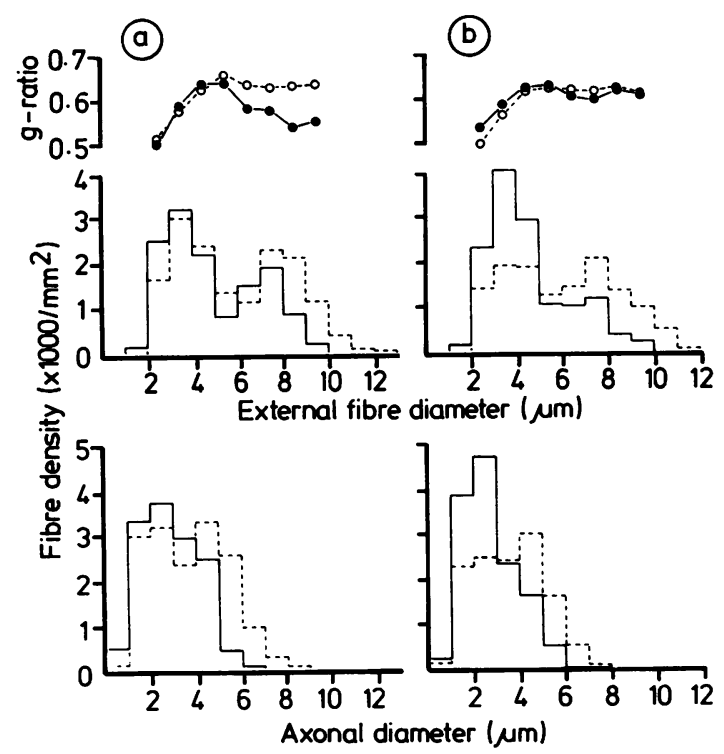

Fig 5 Histograms of axonal and external fibre diameter (expressed as density per $\mathrm{mm}^{2}$ ), and $\mathrm{g}$ ratios for affected and control nerves from one group $A$ and one group $B$ animal. Values for the affected side are shown by continuous lines and filled circles, those for the control side by broken lines and open circles. 450-500 fibres were measured for each nerve. 
Table 2 Axon/external diameter ( $g$ ) ratios for fibres with 7-10 $\mu$ m external diameter

\begin{tabular}{clcl}
\hline & $\begin{array}{l}\text { No of } \\
\text { nerves }\end{array}$ & $\begin{array}{l}\text { No of } \\
\text { fibres }\end{array}$ & $\begin{array}{l}\text { g ratio } \\
\text { (Mean and SD) }\end{array}$ \\
\hline Affected: & & & \\
Group A & 4 & 611 & $* 0.54 \pm 0.07$ \\
Group B & 4 & 291 & $0.62 \pm 0.05$ \\
Control & 6 & 1121 & $0.64 \pm 0.05$ \\
\hline
\end{tabular}

*Significantly different from Group B $(p<0.0001)$ and from controls $(p<0.0001)$; Student's $t$ test.

A nerves, and occasional changes of a similar kind were seen in group B and in control nerves. A simple scoring system was therefore used to grade the severity of the demyelination, and the results are shown in table 3. Group A nerves scored $16 / 21$ for severity, compared with $3 / 21$ for group B nerves and $2 / 33$ for the control nerves from the opposite foot.

Because the asymmetry of the paranodal myelin was similar to that originally described in chronic pressure lesions, ${ }^{4}$ it was concluded that some degree of plantar neuropathy due to pressure on the sole had developed in all the group A nerves, whereas there was only occasional evidence of this in group B or in control nerves.

\section{Discussion}

The use of two groups of operated animals in the present study made it possible to distinguish the effects of tying a contricting ligature for a short period of time, with resulting Wallerian degeneration in many fibres, from the effects of a similar ligature left in place, with the likelihood of persistent changes in fibres which survived the ligature without Wallerian degeneration. The severity of the initial lesion was approximately matched in the two groups by its effect on evoked MAP amplitude and area in response to distal stimulation. Histological studies at the end of the experimental period confirmed that in both groups there had been extensive loss of large myelinated fibres.

In both groups there was an initial fall in MCV distal to the lesion, which is likely to have been due in part to the degeneration of fast-conducting fibres at the time of ligature. Previous experiments on the rabbit have suggested that ligature for 1-2 weeks can itself produce some distal axonal shrinkage which is only slowly reversed. ${ }^{5}$ The aetiology of this early shrinkage is uncertain since an alteration in neurofilament transport ${ }^{6}$ would not be expected to produce such a rapid change. In the guinea-pig there has been no morphological study of distal axonal calibre in the first 2 weeks after ligature, but early shrinkage of the distal axon cannot be excluded as a

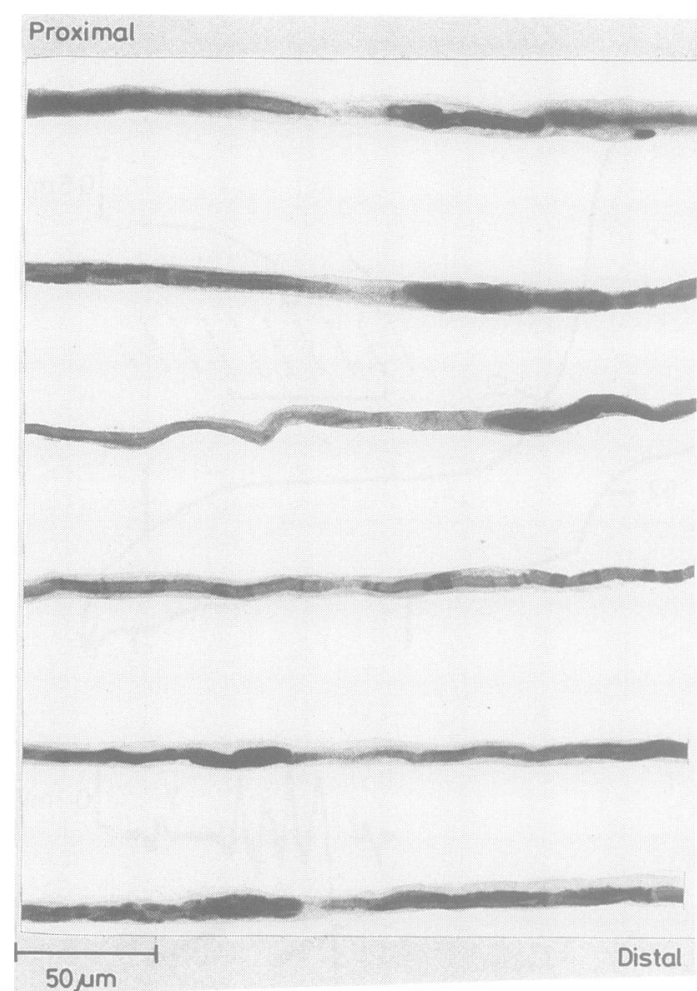

Fig 6 Six consecutive nodal regions of a single myelinated fibre from the affected medial plantar nerve in the mid-foot of a group A animal, to show demyelination and asymmetrical paranodes. In the upper three photographs there is thinning and tapering of the myelin sheath on the proximal side of each node of Ranvier; on the distal side of each node the paranodal myelin is bulky and irregularly folded. The fourth node of Ranvier shows no asymmetry, whereas the 5th and 6th nodes show asymmetry which is the reverse of that seen above.

contributory factor in the reduced MCV seen at 1 and 3 weeks in the present series.

Although there was a later increase in $\mathrm{MCV}$ in group B nerves, this may not indicate recovery from shrinkage, as MCV in control nerves also increased during this period; thus the increase on the affected

Table 3 Severity of local demyelination in plantar nerves

\begin{tabular}{lllll}
\hline & $\begin{array}{l}\text { No of } \\
\text { nerves } \\
\text { examined }\end{array}$ & $\begin{array}{l}\text { No } \\
\text { abnormal }\end{array}$ & Score & $\begin{array}{l}\text { Maximum } \\
\text { possible score }\end{array}$ \\
\hline Group A & 7 & 7 & 16 & 21 \\
Group B & 7 & 2 & 3 & 21 \\
Controls & 11 & 1 & 2 & 33 \\
\hline
\end{tabular}

System of scoring severity: severe $=3$, moderate $=2$, slight $=1$, none $=0$. 
side could have been due to the age-related maturation of surviving motor fibres. Further studies are needed to clarify this point.

In group A, MCV remained low throughout the 9 week observation period after proximal ligature. The mean MCV of $34 \mathrm{~m} / \mathrm{s}$ is similar to that recorded $10-60$ days after proximal ligature in a previous study. ${ }^{1}$ In both series histological examination at the end of the experimental period showed evidence of axonal atrophy in the form of reduced $g$ ratios. In the earlier study the external diameter of single teased fibres was also examined and was found to be reduced in relation to internodal length. In the present study the question may therefore be asked: what was the relationship of the reduced axonal calibre in surviving group A fibres to the failure of recovery of plantar MAP amplitude, and to the development of a local nerve pressure lesion in the sole of the foot?

The distance from the site of ligature in the thigh to the foot muscles was such that reinnervation by regenerating fibres from the level of the ligature occurred late in the experimental period, and did not influence the size of the main deflection of the evoked muscle action potential. Recovery of MAP amplitude and area in group B nerves in the period 5-9 weeks after ligature is therefore likely to have been due to collateral reinnervation by surviving motor axons. The fact that MAP area recovered more completely than amplitude would support this interpretation. The relatively poor recovery of MAP amplitude and area in group A nerves might have been a direct effect of the proximal constriction, since reinnervation after nerve crush is known to be delayed in rabbit tibial nerve distal to a constriction. ${ }^{7}$ However, it appears that an additional local factor was operating in the present experiments, leading to compressive changes in the plantar nerves. These histological changes were present in all seven of the affected group A nerves which were examined, accompanied in five cases by electrophysiological evidence of conduction delay. In contrast, group B nerves and intact unoperated nerves showed only occasional changes.

Pressure neuropathy of the plantar nerves was originally reported in guinea-pigs kept in cages with wiremesh flooring, but not in those kept on solid floors covered in sawdust. ${ }^{3}$ More recent experiments on the rat suggest that plantar neuropathy from the floor of the cage can occur in this species also. ${ }^{8}$ Although the animals in the present experiments were kept on solid flooring covered by a thick layer of sawdust, the electrophysiological findings in our constricted nerves were similar to those described previously in guineapigs kept on wire floors. The histological findings were also indicative of a local compressive lesion in the foot; there was demyelination as well as fresh Wallerian degeneration, accompanied by paranodal asymmetry of a type which has been shown to occur in both animal and human entrapment lesions. ${ }^{4-11}$

It seems unlikely that this compressive lesion in the foot was merely due to paresis of the limb caused by the ligature. While some weakness of dorsiflexion and plantar flexion of the foot and toes was present on testing, there was no visible change in weight-bearing or in the posture of the limb in the cage. Furthermore, the paresis caused by applying the ligature was similar in group B animals; yet in these there was only minimal plantar nerve damage during the period of observation, which did not seem from the physiological and histological findings to be different from that found in control nerves.

It is known that axonal atrophy itself can lead to secondary demyelination and that this process is exaggerated if Schwann cell function is impaired, for example, by a small subclinical dose of diphtheria toxin. $^{1}$ It may therefore be suggested that in the present experiments Schwann cells associated with atrophic axons were unable to maintain compact myelin in the presence of additional mechanical distortion from an external source. It is also likely that the atrophic axons were themselves particularly vulnerable to compression, since Wallerian degeneration in some fibres appeared to commence at the same level as the local demyelination in others. This being so, the failure of recovery of plantar MAP amplitude and area was probably a result of the local plantar nerve lesion, and not a direct effect of the axonal atrophy itself. To refer to this as a possible example of "double crush" or (more correctly) "double entrapment" does not, unfortunately, add to our understanding of the mechanism involved.

The double-crush syndrome was first described by Upton and McComas ${ }^{12}$ to explain the clinical association of median and ulnar neuropathy with cervical spondylosis. It was postulated that pressure on a nerve root might interfere with axonal transport and increase the susceptibility of the distal axon to further compressive damage. It now seems more likely that the clinical association which these authors observed was due to the coexistence of osteoarthritis affecting the cervical spine and the limb joints, with independent nerve lesions at the two levels, ${ }^{13}$ but the concept of double pathology affecting a single nerve fibre is still a useful one. For example, Hopkins and MorganHughes ${ }^{14}$ found that if guinea-pigs were suffering from mild diphtheritic neuritis, they developed localised plantar neuropathy not only on wire mesh flooring but also on solid cage floors, even when these were thickly covered in sawdust. The lesion was only prevented when weight-bearing was avoided altogether by keeping each animal in a sling during the earlier part of the diphtheritic illness. These experimental results were thought to be comparable to the 
development of local nerve damage at entrapment sites in certain human demyelinating neuropathies such as the Guillain-Barré syndrome, diabetes, and tomaculous neuropathy.

In the context of the present experiments one might extend the argument and postulate that conditions such as HMSN 1, uraemic and myeloma neuropathies, in which axonal atrophy is known to occur, ${ }^{15-17}$ could also carry an increased risk of pressure damage to the affected nerves. If this were the case, the present experiments suggest that the result would not be a purely demyelinating lesion but a mixture of demyelination and axonal degeneration.

We thank Mrs A-M Jones for technical assistance. We also thank the National Fund for Research into Crippling Diseases for generous support.

\section{References}

1 Baba M, Gilliatt RW, Harding AE, Reiners $\mathrm{K}$. Demyelination following diphtheria toxin in the presence of axonal atrophy. $J$ Neurol Sci 1984;64:199-211.

2 Baba M, Fowler CJ, Jacobs JM, Gilliatt RW. Changes in peripheral nerve fibres distal to a constriction. $J$ Neurol Sci 1982;54:197-208.

3 Fullerton PM, Gilliatt RW. Pressure neuropathy in the hind foot of the guinea-pig. J Neurol Neurosurg Psychiatry 1967;30:18-25.

4 Ochoa J, Marotte L. The nature of the nerve lesion caused by chronic entrapment in the guinea-pig. $J$ Neurol Sci 1973;19:491-5.

5 Baba M, Gilliatt RW, Jacobs JM. Recovery of distal changes after nerve constriction by a ligature. $J$ Neurol Sci 1983;60:235-46.

6 Hoffman PN, Griffin JW, Price DL. Control of axonal caliber by neurofilament transport. $J$ Cell Biol 1984;99:705-14.

7 Reiners K, Gilliatt RW, Harding AE, O'Neill JH. Regeneration following tibial nerve crush in the rabbit: the effect of proximal constriction. $J$ Neurol Neurosurg Psychiatry 1987;50:6-11.

8 Ortman JA, Sahenk Z, Mendell JR. The experimental production of Renaut bodies in response to mechanical stress. J Neurol Sci 1983;62:233-41.

9 Jefferson D, Eames RA. Subclinical entrapment of the lateral femoral cutaneous nerve: an autopsy study. Muscle \& Nerve 1979;2:145-54.

10 Neary D, Eames RA. The pathology of ulnar nerve compression in man. Neuropathol Appl Neurobiol 1975;1:69-88.

11 Neary D, Ochoa J, Gilliatt RW. Sub-clinical entrapment neuropathy in man. J Neurol Sci 1975;24:283-98.

12 Upton ARM, McComas AJ. The double crush in nerveentrapment syndromes. Lancet 1974;ii:359-61.

13 Frith RW, Litchy WJ. Electrophysiologic abnormalities of peripheral nerves in patients with cervical radiculopathy. Muscle Nerve 1985;8:613.

14 Hopkins AP, Morgan-Hughes JA. The effect of local pressure in diphtheritic neuropathy. $J$ Neurol Neurosurg Psychiatry 1969;32:614-23.

15 Dyck PJ, Johnson WJ, Lambert EH, O'Brien PC. 월 Segmental demyelination secondary to axonaf 음 degeneration in uremic neuropathy. Mayo Clin Pro® 1971;46:400-31.

16 Nukada H, Dyck PJ, Karnes JL. Thin axons relative to myelin spiral length in hereditary motor and sensor neuropathy, type 1. Ann Neurol 1983;14:648-55.

17 Ohi T, Kyle RA, Dyck PJ. Axonal attenuation ans secondary segmental demyelination in myeloma neuropathies. Ann Neurol 1985;17:255-61. 